\title{
Compensation of phonon-induced renormalization of vacuum Rabi splitting in large quantum dots: Towards temperature-stable strong coupling in the solid state with quantum dot-micropillars
}

\author{
C. Hopfmann, ${ }^{1}$ A. Musiał,,${ }^{1,2,{ }^{*}}$ M. Strauß, ${ }^{1}$ A. M. Barth,${ }^{3}$ M. Glässl,${ }^{3}$ A. Vagov, ${ }^{3}$ M. Strauß, ${ }^{4}$ C. Schneider, ${ }^{4}$ \\ S. Höfling, ${ }^{4,5}$ M. Kamp, ${ }^{4}$ V. M. Axt,${ }^{3}$ and S. Reitzenstein ${ }^{1}$ \\ ${ }^{1}$ Institut für Festkörperphysik, Technische Universität Berlin, Hardenbergstraße 36, 10623 Berlin, Germany \\ ${ }^{2}$ Laboratory for Optical Spectroscopy of Nanostructures, Department of Experimental Physics, Faculty of Fundamental Problems of \\ Technology, Wrocław University of Technology, Wybrzeże Wyspiańskiego 27, 50-370 Wroctaw, Poland \\ ${ }^{3}$ Institut für Theoretische Physik III, Universität Bayreuth, 95440 Bayreuth, Germany \\ ${ }^{4}$ Technische Physik, Physikalisches Institut and Wilhelm-Conrad-Röntgen-Research Center for Complex Material Systems, \\ Universität Würzburg, Am Hubland, D-97074 Würzburg, Germany \\ ${ }^{5}$ SUPA, School of Physics and Astronomy, University of St. Andrews, St. Andrews, KY16 9SS, United Kingdom
}

(Received 1 September 2015; published 3 December 2015)

\begin{abstract}
We study experimentally the influence of temperature on the emission characteristics of quantum dotmicropillars in the strong coupling regime of cavity quantum electrodynamics (cQED). In particular, we investigate its impact on the vacuum Rabi splitting (VRS) and we address the important question of the temperature stability of the coherent coupling regime in a semiconductor system, which is relevant in view of both fundamental study and future applications. To study the temperature dependence we investigate an unprecedentedly large number of strong coupling cases (89) in a wide temperature range from 10 up to $50 \mathrm{~K}$, which constitutes a good basis for statistical analysis. The experiment indicates a statistically significant increase of the VRS with temperature in contrast to an expected decrease of the VRS due to the dephasing induced by acoustic phonons. From the theoretical point of view, the phonon-induced renormalization of the VRS is calculated using a real-time path-integral approach for strongly confined quantum dots (QDs), which allows for a numerical exact treatment of the coupling between the QD and a continuum of longitudinal acoustic phonons. The absence of the expected decrease of the VRS with temperature in our experimental data can be attributed to a unique optical property of laterally extended $\mathrm{In}_{0.4} \mathrm{Ga}_{0.6}$ As QDs used in this study. Their electronic structure facilitates an effective temperature-driven increase of the oscillator strength of the excitonic state by up to $40 \%$ in the given temperature range. This leads to enhanced light-matter interaction and overcompensates the phonon-related decrease of the VRS. The observed persistence of strong coupling in the presence of phonon-induced decoherence demonstrates the appealing possibility to counteract detrimental phonon effects in the CQED regime via engineering the electronic structure of QDs.
\end{abstract}

DOI: 10.1103/PhysRevB.92.245403

PACS number(s): 78.67.Hc, 63.20.kk, 78.55.Cr, 42.50.Pq

\section{INTRODUCTION}

The first experimental observations of strong coupling between a single exciton $(\mathrm{X})$ and a single photon realized in semiconductor microcavities [1,2] have triggered enormous research activities in the important field of cavity quantum electrodynamics (cQED). The huge interest in quantum dot (QD)-microcavity systems is motivated by their relevance in both the study on fundamental aspects of quantum optics [3] as well as possible applications in quantum technology. The strong coupling regime is of particular interest because it could pave the way for the implementation of quantum networks [4] or quantum computation schemes [5,6], and to couple its building blocks via flying qubits. In order to implement such complex systems relying on the strong coupling regime, it is crucial that the light-matter interaction rate exceeds dissipative loss rates, which puts stringent requirements on the device technology. At low temperatures cavity photon losses are still the main source of dissipation, despite the significant optimization of fabrication techniques which has led to a strong increase of the quality $(\mathrm{Q})$ factors of microand nanocavities $[7,8]$. The situation can change significantly

*anna.musial@physik.tu-berlin.de at elevated temperatures when phonon-related dissipation sets in, which is of particular importance for possible applications. In fact, a strong influence of phonons on cQED phenomena has been predicted theoretically [9-16], but the few existing experimental observations are rather limited [17-19].

In this work we focus on investigating the influence of temperature on the vacuum Rabi splitting (VRS) in a strongly coupled QD-microcavity system. The experimental studies have been performed on high-Q low-mode volume micropillar cavities with a single layer of self-assembled $\mathrm{In}_{0.4} \mathrm{Ga}_{0.6}$ As QDs as the active medium. Within a statistical approach, 89 strong coupling cases with different resonance temperatures have been studied to establish the temperature dependence of the VRS. It should be noted that the temperature range from 10 to $50 \mathrm{~K}$ covered by our investigation of coupled QD-micropillars is by a factor of 4 larger than in a previous study on a single QD-nanocavity system by Ota et al. [17].

The obtained experimental results were compared and contrasted with a model of the QD-micropillar accounting for the influence of acoustic phonons and photon losses on the VRS in the case of strongly confined QDs. To this end we used a hybrid approach combining a numerically exact microscopic calculation of phonon-related pure dephasing [20] with a phenomenological treatment of the cavity photon losses [21]. We thus present a comprehensive theoretical study 
on the influence of the properties of both the emitter as well as of the cavity (QD size, cavity Q-factor, and lightmatter coupling constant-g) on the vacuum Rabi splitting renormalization.

The paper is organized as follows: Sec. II introduces the investigated structures and the experimental setup. Section III describes and justifies the applied statistical approach. In Sec. IV the experimental results are presented. Section V addresses theoretically the VRS behavior under influence of acoustic phonons and comprises a comparison with the experimental results. Section VI concludes the paper.

\section{EXPERIMENT}

The systems under study are QD-micropillar cavities. The planar microcavity consists of a GaAs $\lambda$ cavity with high reflectivity AlAs/GaAs distributed Bragg reflectors (DBRs)26 (30) $\lambda / 4$ pairs on the top (bottom) side, respectively. The micropillars are defined by high-resolution electron beam lithography and patterned by plasma etching of the planar microcavity [7]. In this way high Q-factor (13 000 on average) microcavities with a nominal diameter of $1.8 \mu \mathrm{m}$ (mode volume: $0.43 \mu \mathrm{m}^{3}$ or $10\left(\frac{\lambda}{n}\right)^{3}$, where $\lambda$ is a cavity resonance wavelength in vacuum and $n$ is the effective refractive index of the cavity material), are fabricated. The active medium consists of a single layer of large $\mathrm{In}_{0.4} \mathrm{Ga}_{0.6} \mathrm{As}$ QDs featuring a more than a factor of 2 higher oscillator strength (OS) if compared to smaller strongly confined QDs in the same material system [1,22-25]. To differentiate the QDs investigated here from typical strongly confined QDs we refer to them as "large" QDs. The typical dimensions of these nanostructures are: (20-30) $\mathrm{nm}$ in width, (20-50) $\mathrm{nm}$ in length, and (5-7) $\mathrm{nm}$ in the vertical direction (including wetting layer). The increase in the lateral size in the strain-based Stranski-Krastanow growth mode was possible due to lowering the strain via decreasing the amount of indium in the InGaAs alloy compound [26]. This has been proven to be a very effective method of enhancing the oscillator strength of QD transitions, leading to the observation of the strong coupling in the single $\mathrm{X}$-single photon regime in QD-micropillar cavities [1]. It turns out that the structures described above are a perfect choice for our statistical study on the temperature influence on VRS in a wide temperature range since up to $50 \%$ of the investigated QD-micropillars allow for the observation of strong coupling.

The spectroscopic studies were performed by means of high-resolution microphotoluminescence ( $\mu \mathrm{PL})$ spectroscopy. The QD-micropillars were excited nonresonantly with a frequency doubled $\mathrm{Nd}$ :YAG laser at $532 \mathrm{~nm}$ operating in continuous wave mode. The measurements were performed in the low excitation regime (typical excitation power: $10 \mu \mathrm{W}$ ), i.e., well below saturation of the QD X, to prevent excitation power-induced dephasing of the excitonic system. The excitation was focused on the chosen micropillar via a long working-distance $(20 \mathrm{~mm})$ microscope objective $(0.4$ numerical aperture) which is simultaneously used for the detection. The spatial resolution of the optical setup is about $3 \mu \mathrm{m}$, which enables addressing individual micropillarsthe distance between adjacent micropillars is $10 \mu \mathrm{m}$. The sample was mounted in a continuous-flow helium cryostat enabling temperature variation in the range of 5-340 K. The emission was spectrally resolved using a 0.75-m-focal-length spectrometer equipped with a liquid nitrogen-cooled $\mathrm{Si}-\mathrm{CCD}$ detector providing an overall spectral resolution of about $25 \mu \mathrm{eV}$.

\section{EXPERIMENTAL METHODOLOGY: STATISTICAL APPROACH}

Previous experimental reports on the temperature dependence of the Rabi splitting were restricted to low temperatures below $15 \mathrm{~K}$, very narrow temperature ranges (12 K at most) $[17,19]$ and standard strongly confined InAs QDs. In the low temperature regime (below $10 \mathrm{~K}$ ) the dominating effect is the influence of the imbalance between phonon absorption- and emission-assisted processes on off-resonant QD-cavity coupling. Additionally, a monotonic decrease of VRS up to $14.8 \mathrm{~K}$ attributed to the phonon-induced reduction of the light-matter coupling strength was observed. Up till now the latter has not been investigated experimentally at higher temperatures. In Ref. [19] the Rabi splitting of a coherently driven QD has been investigated and the decrease of the Mollow-triplet sidebands splitting with increasing temperature was observed and attributed to phonon-induced renormalization of the Rabi splitting. This study was also limited to temperatures below $15 \mathrm{~K}$ and without involving cavity effects. Theoretical reports in the high temperature regime predict a decreasing tendency in renormalized VRS as a function of temperature (for 10, 50 , and $100 \mathrm{~K}$ ) in the case of $g$ values typical for coupled QD-microcavity systems and a reversed trend for much larger coupling strength-typical for laser-driven QDs [21].

Hereby we present an alternative, statistical approach to investigate the influence of the temperature on the emission characteristics of a strongly coupled QD-microcavity system. The comparatively high surface QD density of $10^{10} / \mathrm{cm}^{2}$ leads to a spectral density of approximately five single QD emission lines per meV near the fundamental cavity mode (CM) of a micropillar with $d_{c}=1.8 \mu \mathrm{m}$ diameter. For this reason and because of high sample quality, we find on average two Xs per micropillar coupling strongly to the fundamental CM which constitutes a good basis for our statistical approach. We used temperature as a tuning parameter to shift the single QD X emission line into resonance with the fundamental CM [Fig. 1(a)]. Therefore, we are limited to QDs with a spectral separation below $1 \mathrm{meV}$ from the $\mathrm{CM}$ and are thus probing only a very small subset of the whole QD ensemble which shows an inhomogeneous broadening of $62 \mathrm{meV}$ [26]. As a consequence the investigated QDs are similar in both their vertical size and their In content, which are the crucial factors determining the emission energy. Due to spectral differences in the initial (low-temperature $10 \mathrm{~K}$ ) separation between the $\mathrm{CM}$ and the $\mathrm{X}$ transition energy, the resonance conditions are reached for various temperatures. For each QD-micropillar, the vacuum Rabi splitting and the resonance temperature can be determined (we restricted our study to perfect spectral matching, i.e., zero detuning between the energy of the $\mathrm{X}$ transition and the $\mathrm{CM}$ ). Therefore, a comprehensive examination of many strong coupling cases should allow for an observation of the temperature dependence of the VRS. The essential condition for this approach to be successful is that the scattering of the experimental data related 

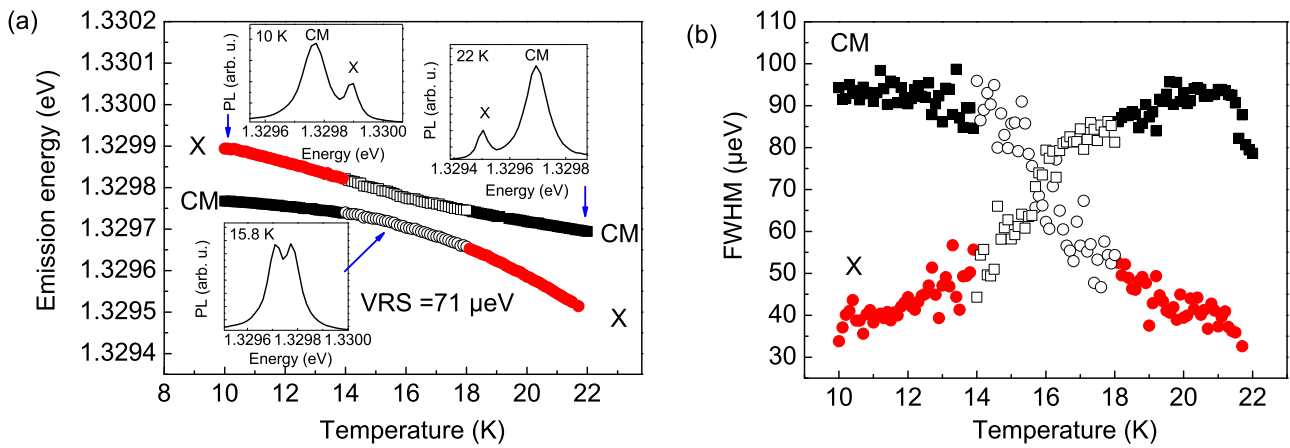

FIG. 1. (Color online) Experimental data for a strongly coupled single $\operatorname{~n}_{0.4} \mathrm{Ga}_{0.6} \mathrm{As}$ QD X and a CM of a $1.8 \mu \mathrm{m}$ diameter micropillar exhibiting a VRS of $71 \mu \mathrm{eV}$. (a) Emission energy dependence of the QD X (red dots) and the CM (black squares) on the temperature with characteristic anticrossing on resonance (coupling regime indicated by open symbols) with insets showing exemplary spectra for different detunings between the CM and the QD X (10, 15.8, and $22 \mathrm{~K}$ ). (b) Temperature dependence of the QD X (red dots) and the CM (black squares) linewidth with characteristic crossing on resonance with the coupling regime indicated by open symbols.

to individual differences between the investigated systems is smaller than the temperature influence. We have good evidence that this is the case for the chosen QD-micropillar system, which will be explained further in this section.

Before we discuss our experimental findings, we first introduce all the factors that can lead to the differences in VRS between various QD-micropillar systems at a given temperature (equal resonance temperatures). If the strongly coupled QD microcavity is simply described as a system of two coupled oscillators and the presence of phonon-induced dephasing is not taken into account, the on-resonance splitting between the two modes depends on the light-matter coupling constant $g$ and the cavity- and exciton-related losses described by the respective linewidths $\gamma_{C}$ and $\gamma_{X}$, and is given by [1]

$$
\Delta E=2 \sqrt{g^{2}-\frac{\left(\gamma_{C}-\gamma_{X}\right)^{2}}{16}} .
$$

However, for our present systems the homogeneous (lifetime-limited) broadening of the excitonic line $\gamma_{X}$, which at low temperatures is in the range of a few $\mu \mathrm{eV}$ reflecting the relatively short X lifetime of about 350 ps [22,27], is negligible in comparison to the cavity linewidth $\gamma_{C}$, which is up to two orders of magnitude larger for an average Q factor of 13000 (Fig. 3). In the data post-processing we can restrict ourselves to the pillars exhibiting Q-factors within a specified range to post-select only strong coupling cases in which CM featuring similar $\gamma_{C}$ are involved, which allows our ensemble to be homogeneous in terms of this loss mechanism. Additionally, the light-matter coupling constant $g$ itself may vary between the different strong coupling cases due to various properties of the QD-micropillar system, which we shall discuss now. The coupling constant depends on both QD and cavity properties:

$$
g_{\max } \propto \sqrt{\frac{f_{\text {osc }}}{V_{m}}},
$$

where $f_{\text {osc }}$ is the oscillator strength of the $\mathrm{X}$ transition and $V_{m}$ is the CM volume. A possible variation of $V_{m}$ can be neglected as the study is restricted to only one pillar size. A nominal diameter of $d c=1.8 \mu \mathrm{m}$ has been chosen as optimal for the realization of strong coupling because pillars with this diameter exhibit a maximum $\mathrm{Q} / d_{c}$ ratio which is the technological figure of merit for the observation of strong coupling [1]. Due to negligible variations of the mode volume, the light-matter coupling strength is therefore determined by the oscillator strength. In the case of large QDs the oscillator strength is governed mainly by the in-plane size of the QD [28] which, according to structural data, varies in the range of (20-30) $\mathrm{nm}$ in width and (20-50) $\mathrm{nm}$ in length. This variation results from a local strain at the position where the QD is formed, which is in our case determined by the In concentration. Our previous magneto-optical study [22] on a series of $\operatorname{In}_{x} \mathrm{Ga}_{1-x} \mathrm{As}$ QD structures showed that increasing the In content from 0.3 to 0.6 leads to a reduction of the mean lateral extension of the wave function from 15 to $7 \mathrm{~nm}$ and consequently the OS is also reduced from 50 to 10 . This indicates that a significant variation of the In content is needed to affect the OS. Therefore, the question arises how strongly the In concentration varies within the ensemble of QDs under study. For the structures used here the emission energy can be used as an indicator for the In concentration, because it has been shown that a variation of the In content leads to a significant change of the QD transition energy [29], which can even overcome the influence of the variation in QD height. Most importantly, this means that the OS and light-matter coupling strength is expected to be similar for all studied QDs.

Beyond the simple approximation of Eq. (1), different QD-micropillar systems can be subjected to different phononinduced dephasing leading to different renormalization of the VRS at a given temperature. The strength of the Xphonon coupling is typically determined by the size of the smallest extension of the electronic wave function, because this corresponds to the largest contribution of phonon modes in $k$ space [30,31]. As we discussed above, the relevant QD height distribution is expected to be very narrow and therefore also the phonon effects are expected to be similar for all QDs in the probed ensemble.

So far our analysis was restricted to the case of the QD being perfectly matched to the CM. Deviations from this ideal situation will cause a lowering of the coupling constant 
which can lead to further differences between the investigated systems. The spectral mismatch (detuning) is not relevant for our discussion, because we limit ourselves to QDs in resonance with the CM. The light-matter coupling is also affected by the dipole direction relative to the electric field as well as by the position of the QD with respect to the center of the cavity, i.e., the distance of the QD to the antinode of the electromagnetic field distribution of the fundamental CM. This is reflected by the formula $g \propto \boldsymbol{d} \boldsymbol{E}$, i.e., the light-matter coupling constant is proportional to the dot product between the dipole moment of the QD transition $\boldsymbol{d}$ as well as the electric field $\boldsymbol{E}$ at the emitter location. As far as the linear polarization directions are concerned, we can, within a first approximation, assume that the QD polarization axes are elongated in crystallographic [1-10] and [110] directions due to the preferential elongation of the nanostructures in [1-10] direction, as proved by structural studies [26]. On the other hand, the polarization of the cavity modes is related to a slight unintentional deviation from the circular cross section which lifts the degeneracy of the two fundamental modes. The nondegenerate modes are linearly polarized perpendicular to each other and tilted on average by $45 \mathrm{deg}$ with respect to the QD elongation direction [7,32]. Even though distinguished optical axes are present in our system, local strain and piezoelectric field distributions can still lead to deviations from the crystallographic directions described above. The largest deviations observed in the case of self-assembled QDs without rotational symmetry are up to $20^{\circ}$ [33], which still translates into only $6 \%$ variation in the coupling constant. On the other hand, the spatial mismatch between the QD and the CM can vary in a broad range from the QD being located in the very center of the micropillar and at its edge. The maximal coupling constant expected in the investigated micropillars for a QD perfectly matched to the CM equals $56 \mu \mathrm{eV}$ [1]. The minimal coupling constant relevant in our case is related to the transition to the weak coupling regime. A simple estimation based on Eq. (1) for a typical cavity linewidth of $100 \mu \mathrm{eV}$ gives $g_{\min } \approx \frac{\gamma_{C}}{4} \approx 25 \mu \mathrm{eV}$, which translates into a difference of almost $50 \%$ in the coupling constant. This brings us to the conclusion that the random spatial mismatch between the QD position and the antinode of the electromagnetic field distribution at the center of the cavity will most probably be the main source for scattering of the measured VRS in our set of experimental data points for a given temperature. Assuming that the QD distribution within the cavity is spatially homogeneous on the scale of the micropillar cross-section area, the distribution of the light-matter coupling constants $g$ for a given temperature is therefore expected to be alike the electric field $E$ radial distribution of the fundamental $\mathrm{HE}_{11} \mathrm{CM}$ described by the Bessel function $J[0, r]$ [34] weighted with the probability of the QD being located at a certain distance from the micropillar center. In the ideal case of an isolated system without any kind of losses, this would also be reflected in a similar distribution of the VRS according to Eq. (1). However, as argued above, imperfect matching of the QD to the CM is not expected to lead to a systematic temperature dependence.

Besides temperature-independent changes of the VRS due to the different properties of the strong coupling cases with the same resonance temperature, the temperature itself influences the physics of a single QD-micropillar system in a way that affects the VRS. For example an increased temperature leads to stronger phonon effects, and it is well known that this implies a distinct decrease of the VRS with rising temperature, which is in sharp contrast to the previously described unsystematic temperature-independent scattering when looking at different strong coupling cases. On the other hand, the maximal VRS for a given temperature should certainly correspond to QDs that are spatially matched to the CM. As a result, looking at the envelope function of the experimental results, i.e., extracting the maximum VRS observed for each temperature, should allow us to extract the temperature-dependent behavior independently of the possible differences between the investigated QD-micropillar systems.

\section{EXPERIMENTAL RESULTS AND DISCUSSION}

The proposed statistical approach has been utilized to experimentally study the influence of phonons on the vacuum Rabi splitting. A large and statistically significant number (89) of strong coupling cases from a single sample was experimentally studied by temperature tuning and the respective resonance temperatures were determined based on the energy vs temperature dependence [cf. Fig. 1(a)]. Each of the strong coupling cases was verified by the observation of the characteristic mode anticrossing in the energy dispersion as well as by crossing in the linewidth dependence on temperature [Fig. 1(b)], indicating an exchange in character between the $\mathrm{CM}$ and the $\mathrm{X}$ and the formation of polaritonic states on resonance. The results of the measurements are summed up in Fig. 2 where the extracted VRS is presented as a function of the resonance temperature. At first glance no clear tendency can be observed due to the scattering of the experimental data, which can be attributed predominantly to the varying QD positions

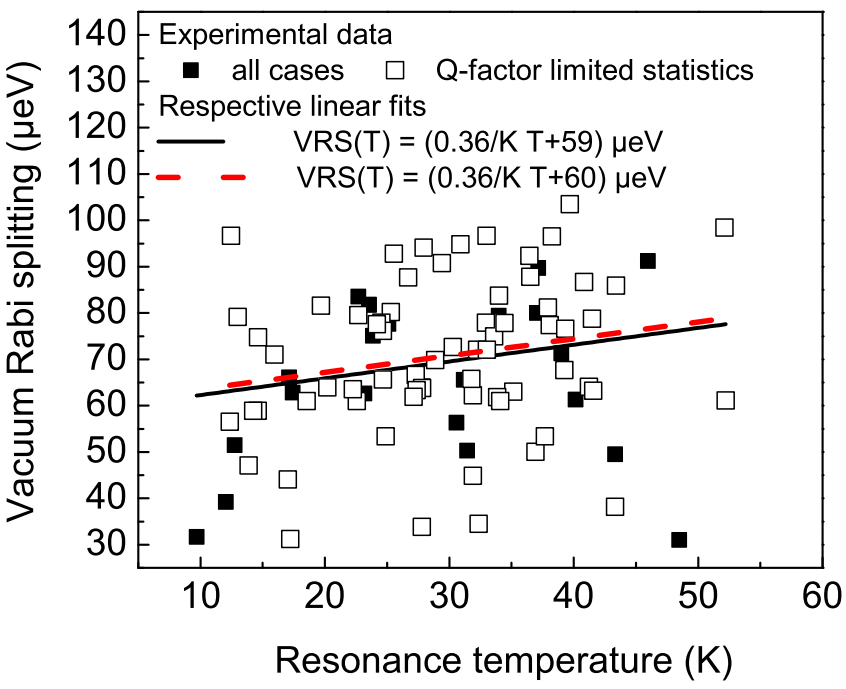

FIG. 2. (Color online) VRS as a function of the resonance temperature for all (89) measured strong QD-micropillar CM coupling cases (black squares) and for cases with Q-factors in the range of 11000-15000 (empty symbols) with respective linear fits-black solid line for full statistics and red dashed line for the Q-factor limited statistics. Each experimental data point represents a strong coupling case for a different QD-micropillar system. 


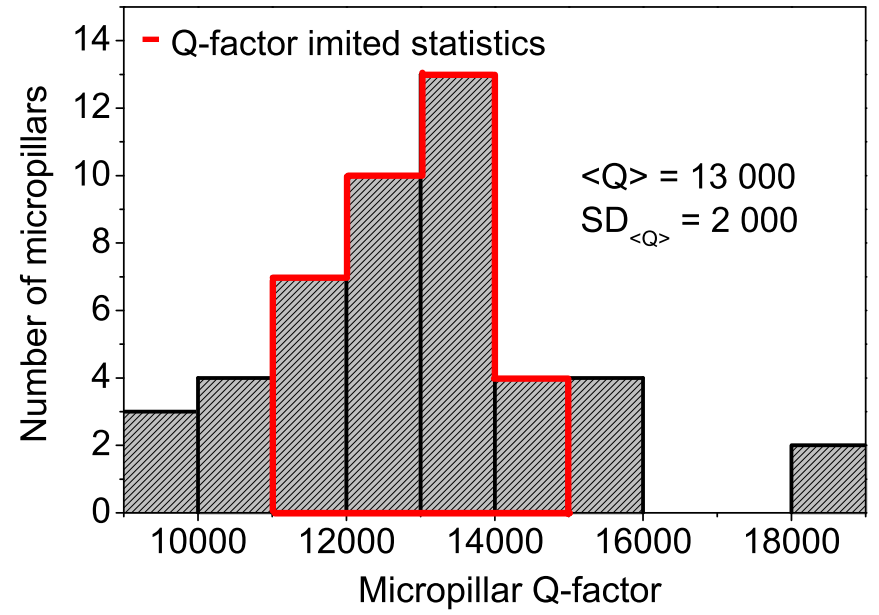

FIG. 3. (Color online) The histogram of Q-factors for investigated micropillar cavities with the range of Q-factors used in the post-selection process for Q-factor limited statistics marked in red.

with respect to the maximum of the mode field distribution in the systems under study. For the same reason, the envelope of the measured dependence is not well defined. The simplest least-squares linear fit to the experimental data (solid black line) exhibits a slope of $0.36 \mu \mathrm{eV} / \mathrm{K}$, but a quantitative statistical analysis is needed to evaluate the significance of this tendency. We performed a standard analysis of variance using a statistical F-test to evaluate the goodness of the fit with the null hypothesis being that there is no tendency in the experimental data (which would be the case if the VRS is constant as a function of the temperature). This would mean that the slope of the linear fit is a result of random scattering of the experimental data. The $p$ value calculated for the experimental data equals 0.049 , which means that the probability that the null hypothesis is true is as low as $4.9 \%$. While for strongly confined QDs one would expect a temperature-dependent decrease of the VRS due to the increasing phonon induced dephasing of the system, we find that for the large QDs studied here there is a $95.1 \%$ probability that the experimental data shows an increasing trend. To exclude possible variations of the VRS due to different cavity $\mathrm{Q}$ factors we employed a post-selection scheme and restricted the data to micropillars with $\mathrm{Q}$ factors within one standard deviation of 2000 from the mean value of 13000 (Fig. 3). Such a limited statistics is presented in Fig. 2 (empty symbols). The slope of the linear fit to the experimental data $(0.36 \mu \mathrm{eV} / \mathrm{K})$ does not change in comparison to the full statistics (Fig. 2 red dashed line) and the probability that the trend is increasing is equal to $90.1 \%$. This supports the hypothesis that there is an additional effect that counteracts the temperature-dependent phonon-induced decrease of the VRS, which was not observed in the case of strongly confined QDs.

Naturally the question arises what physical reason can lead to such an increase of the VRS as a function of temperature, counteracting the phonon influence. The differences between the QD-micropillars can only lead to a substantial scattering of the experimental data for a given temperature as was described in the previous section, but not to an experimentally observed distinct dependence. In particular, one cannot explain the increase of the VRS if all the relevant parameters influencing the splitting are scattering randomly for the different QD micropillars. Thus, to explain the experimentally observed positive trend, we either have to conclude that the commonly expected phonon-induced decrease of the VRS with temperature does not apply to our systems or one of the parameters exhibits a temperature dependence that we did not consider so far. The strength of the phonon coupling depends on the deformation potential constants and the extension of the wave function in the direction of the strongest confinement (the growth direction in our case) - for neither of these parameters has a temperature dependence been reported so far, nor is it expected. Moreover, Eq. (1) indicates that the VRS depends on the losses in the system and the light-matter coupling constant. The properties of the cavity are only slightly affected by the temperature. The length of the cavity changes due to thermal expansion and the refractive index of the cavity material is modified which slightly changes the resonance frequency of the cavity as can be seen in Fig. 1, but the cavity losses are not temperature dependent. On the other hand, the excitonic losses increase with temperature due to the activation of nonradiative recombination channels as well as increased pure dephasing due to acoustic phonons [35-39]. The increase of the $\mathrm{X}$ homogeneous linewidth translates into a decrease of the second term in Eq. (1) which can indeed result in the increase of the Rabi frequency with temperature. The strength of this effect can be estimated based on the measurements of the temperature-induced changes in the homogeneous linewidth performed for QDs in the same material system [35]. One has to keep in mind that the QDs in this reference paper are smaller than the investigated ones and stronger dephasing is expected for smaller QDs, which means that the estimated effect would be the absolute upper limit for our case. Bayer et al. reported an increase of the homogeneous linewidth from 2 up to $30 \mu \mathrm{eV}$ at $60 \mathrm{~K}$. Assuming a constant cavity linewidth of $100 \mu \mathrm{eV}$ typical for our experiments, this would only lead to a change in the VRS below $2 \%$, which is much weaker than the predicted phonon-induced renormalization. As far as the light-matter coupling constant is taken into account, we have to consider the oscillator strength of the $X$ transition and the mode volume [Eq. (2)]. As discussed above, the mode volume is not altered significantly with temperature, but the oscillator strength which is determined by the overlap of the electron and hole wave functions as well as the wave function extension [40] might in principle be temperature dependent. In fact, a previous study on larger QDs in the same material system $\operatorname{In}_{0.3} \mathrm{Ga}_{0.7} \mathrm{As} / \mathrm{GaAs}$ proved that the wave function extension of the state from which the emission takes place can actually undergo changes [29] that can be attributed to a temperature-induced carrier redistribution within the closely spaced electronic sublevels related to possible confining potential fluctuations within large QDs. Similar effects have been observed in the case of quantum dashes [41] featuring an even larger nanostructure volume in the range of $4.5 \times 20-30 \times 100-150 \mathrm{~nm}^{3}$. The strength of this effect can be estimated based on available magneto-optical experimental data [29]. As the QDs in the above reference are larger than the ones investigated in this work, this estimation will constitute an upper limit of the expected changes. The lateral mean wave function extension $2 \sqrt{\left\langle\rho_{X}^{2}\right\rangle}$ can be experimentally 


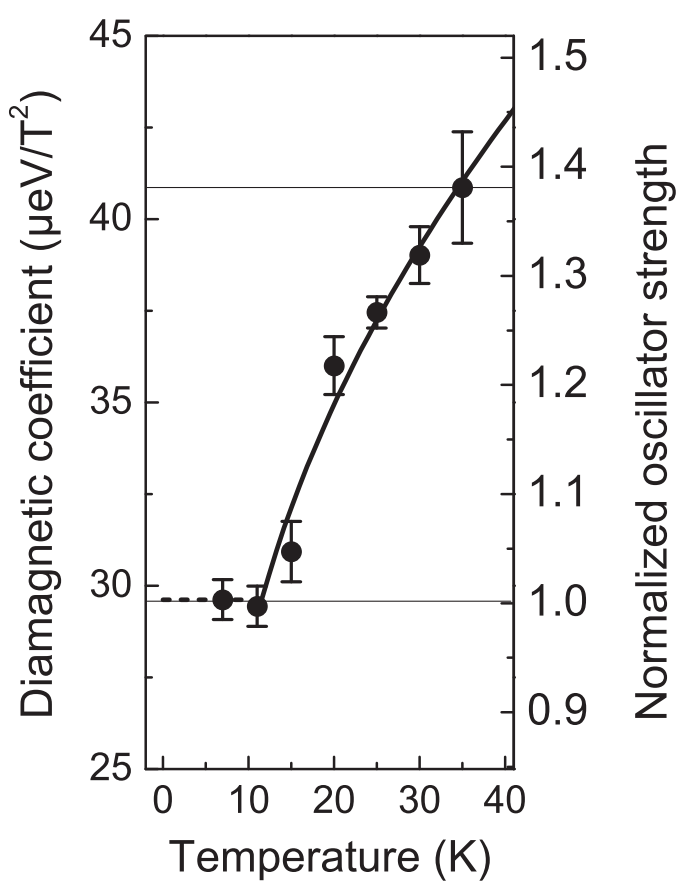

FIG. 4. Experimentally obtained temperature dependence of the diamagnetic coefficient (left axis) for a single $\operatorname{In}_{0.3} \mathrm{Ga}_{0.7} \mathrm{As}$ QD (from Ref. [29]) and oscillator strength normalized to its low-temperature value (right axis) determined based on this measurements. The black line represents a square root fit to experimental data for $T>11 \mathrm{~K}$ and constant value for $T<11 \mathrm{~K}$.

probed via the diamagnetic coefficient $\kappa$ according to the relation

$$
2 \sqrt{\left\langle\rho_{X}^{2}\right\rangle}=\frac{2 \sqrt{8 \mu \kappa}}{e},
$$

where $\mu$ is the reduced effective mass of the exciton and $e$ is the elementary charge. This means that the temperature-induced change of the diamagnetic coefficient from $(29.6 \pm 0.6)$ to $(40.9 \pm 1.5) \mu \mathrm{eV} / \mathrm{T}^{2}$ (as shown in Fig. 4 for an exemplary single QD-QD1 from Ref. [29]) corresponds to a change of the lateral wave function extension from $(15.7 \pm 0.8)$ to $(18.4 \pm$ 2.2) $\mathrm{nm}$. This increase of the wave function extension by a factor of 1.17 in the temperature range from 7 to $35 \mathrm{~K}$ (Fig. 4), translates into a similar increase of the light-matter coupling constant through its square root dependence on the oscillator strength [Eq. (2)]. In the case of an isolated system without losses $\left(\gamma_{C}=\gamma_{X}=0\right)$ also the VRS should increase by a factor of 1.17. This effect provides a possible qualitative explanation of the experimentally observed tendency of an increasing VRS as a function of temperature.

\section{THEORETICAL RESULTS AND COMPARISON WITH THE EXPERIMENT}

In this section the modeling of a strongly coupled QDmicrocavity system is presented and the phonon influence on the coherent dynamics in terms of the VRS is discussed. The QD is modeled as an electronic two-level system consisting of the ground state and an $\mathrm{X}$ state, which are resonantly coupled to a single quantized $\mathrm{CM}$ making the details of the cavity design and absolute emission energy irrelevant. The Jaynes-Cummings type light-matter interaction preserves the excitation number of the system, which therefore can be restricted to the $\mathrm{X}$ state without cavity photons and the QD ground state with a single photon. However, because the Jaynes-Cummings model does not account for cavity losses it has to be extended accordingly, as will be explained further below. Furthermore, we include the coupling of the excitonic system to a continuum of longitudinal (bulk) acoustic phonons via the deformation potential, which is the dominant coupling mechanism in nonpolar materials without permanent dipole moments. We assume the QD confinement potential to be spherically symmetric and adjusted to the expected vertical dimension of the QDs in the sample, which is justified because this is the direction of the strongest confinement of the QD and is therefore responsible for the restriction of the phonon wavelengths, which can effectively couple to the electronic system. Furthermore, we assume electrons and holes being in the harmonic ground state implying Gaussian wave functions. Material specific effective mass differences are included and translate into a fixed ratio between the localization lengths of the electron and hole. Therefore, in this model the spatial extension of the electron wave function, which we refer to as the QD size, is the only parameter determining the strength of the X-phonon coupling besides material parameters, which are the same for all QDs in the sample such as the deformation potential constants, mass density, etc. More details of the described model are given in Ref. [12]. It should be noted that this kind of modeling has been proven to quite accurately describe the dynamics of self-assembled InGaAs/GaAs QDs observed experimentally [42-44]. To calculate the dynamics of this coupled system we use a previously developed real-time path-integral approach $[12,20]$, which is especially suitable, because it allows for treating the carrier-phonon coupling in a numerically exact way. As we pointed out earlier, besides the coupling to phonons, also cavity losses have to be included in the model due to the different Q-factors of the micropillars. However, within the path-integral approach this is very challenging and might not be practical, because of the increased number of paths required when taking into account more states with different photon numbers. Instead, the cavity losses can be described within a hybrid approach proposed in [21] which uses the exact results of the microscopic model of phonon-induced pure dephasing described so far as input parameters for a phenomenological rate model describing the remaining loss channels (cavity losses and radiative decay) within the Lindblad formalism.

The results of our calculations for the phonon-induced renormalization of the VRS are presented in Fig. 5. It shows the temperature dependence of the VRS for two different values of the light-matter coupling constant of $56 \mu \mathrm{eV}$ (the maximum value expected in the investigated QD-micropillar ensemble) and $25 \mu \mathrm{eV}$ (the minimal coupling constant for which the strong coupling regime is maintained) and various QD sizes, respectively. These calculations have been performed without taking photon losses into account. The presented results show that the phonon coupling leads to a strong decrease (down to $50 \%$ ) of the VRS in the experimentally accessible temperature range of 5-60 K. The decrease is linear for low temperatures (up to $20 \mathrm{~K}$ ) and followed by sublinear behavior resulting 


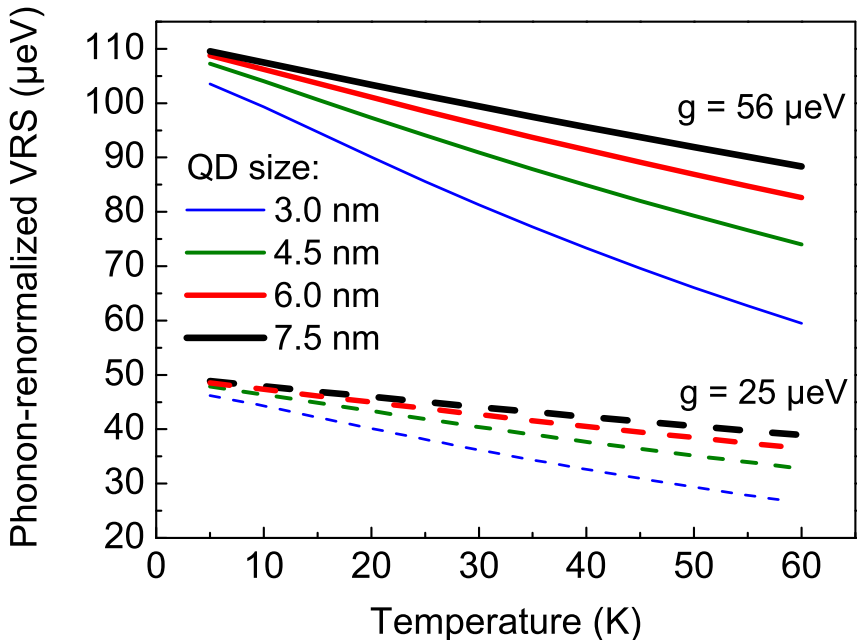

FIG. 5. (Color online) Calculated acoustic phonon-induced temperature dependence of the VRS for two light-matter coupling constants of $25 \mu \mathrm{eV}$ (dashed lines) and $56 \mu \mathrm{eV}$ (solid lines) as well as for various QD sizes: $3.0 \mathrm{~nm}$ (blue, the thinnest line), $4.5 \mathrm{~nm}$ (green), $6.0 \mathrm{~nm}$ (red), and $7.5 \mathrm{~nm}$ (black, the thickest line).

from the subsequent occupation of all phonon states that can effectively couple to the electronic system and an equalization of the probability for phonon emission- and absorptionassisted processes at elevated temperatures. The decrease is stronger for smaller QDs. The light-matter coupling constant slightly changes the slope of the observed dependences, but most importantly it changes the initial (low-temperature) value of the VRS by even more than a factor of 2. This is very important for the comparison with the experiment, because the phonon-free case is not accessible experimentally and the value of the light-matter coupling constant would have a striking effect on the observed dependence and as a result would be the most important source of scattering in the experimental data.

In the next step, another possible difference between individual QD-micropillars, namely the photonic losses described by means of the Q-factor, are included. In Fig. 6 the Q-factor is varied in the experimentally relevant range from 9000 to 18000 (Fig. 3) for two extreme values of the light-matter coupling constant ( 25 and $56 \mu \mathrm{eV}$ ). For large $g$, the Q-factor variation introduces a very slight change of the slope of the VRS dependence on temperature, which is more pronounced for larger $\mathrm{Q}$ factors. The $\mathrm{Q}$ factor also influences the initial value of the VRS and, in the extreme case, i.e., for $Q=9000$, can change it by $25 \%$, which is on a similar scale as the impact of a variation of the coupling constant $g$. Looking at the low $g$ case, the decrease of the Q-factor can lead to a transition from the strong to the weak coupling regime when the losses overcome the coupling strength and the cavity is not able to store the photon for long enough (indicated by a VRS equal to 0 in Fig. 6).

All previous considerations predict a decreasing trend in the VRS dependence on temperature which cannot be clearly seen in the experimental results. However, modeling a possible temperature-induced redistribution of the carriers over several electronic sublevels related to confining potential fluctuations that might lead to higher OS, microscopically

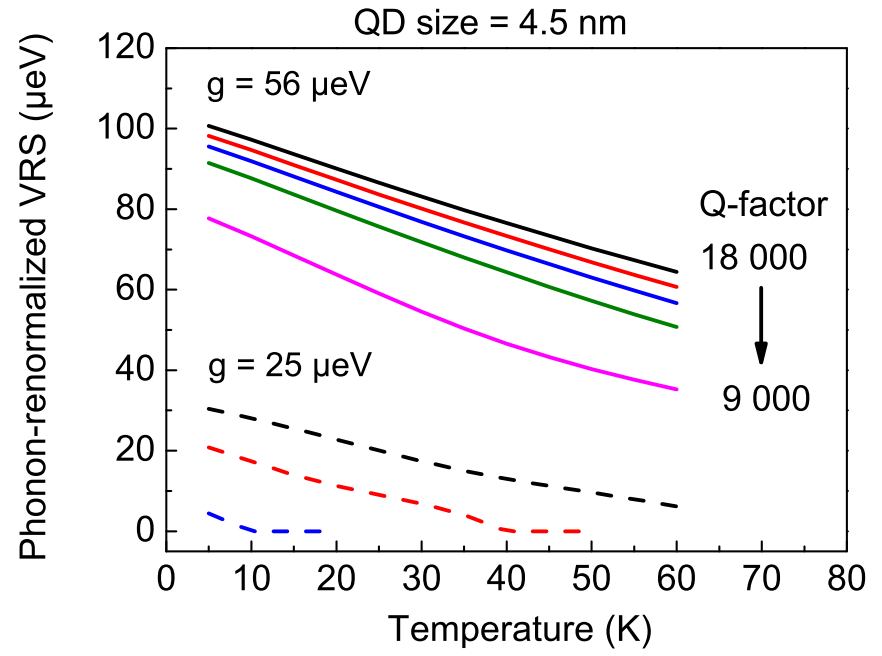

FIG. 6. (Color online) Calculated acoustic phonon-induced temperature dependence of the VRS of a $4.5 \mathrm{~nm}$ QD for two light-matter coupling constants of $25 \mu \mathrm{eV}$ (dashed lines) and $56 \mu \mathrm{eV}$ (solid lines) as well as for various cavity Q-factors in the experimentally relevant range from 9000 to 18000 .

is clearly beyond the scope of this work. It cannot simply be included in the path-integral calculations as extending the involved electronic states beyond the two-level system is already very challenging and models with more than four levels have not been addressed so far. However, the effect can be addressed in the calculations in a phenomenological way by considering a temperature dependence of the light-matter coupling constant based on a fit to the experimental data shown in Fig. 4 taken from Ref. [29]. The OS and its dependence on temperature have a complex microscopic origin and cannot be described analytically. In the present case, the $f_{\text {osc }}(T)$ dependence can be described phenomenologically to a good approximation by a square root dependence reproducing well the data in Fig. 4 above $10 \mathrm{~K}$. The associated light-matter coupling constant is calculated according to Eq. (2) assuming that the light-matter coupling constant at low temperature $g(T=7 \mathrm{~K})=g_{\max }=56 \mu \mathrm{eV}$, where $g_{\max }$ is the maximal achievable value estimated for investigated QD-micropillars. For temperatures exceeding $35 \mathrm{~K}$ (i.e., beyond the available experimental data) we estimated a lower and upper bound of the $f_{\text {osc }}(T)$ dependence and extracted the relevant $g(T)$ to use it as an input for the calculations of the VRS vs temperature for the two extreme cases. As a lower limit, $g$ is assumed to be constant and equal to the $g$ value for $35 \mathrm{~K}$. This estimation is based on the assumption that the light-matter coupling constant does not increase substantially for $T>35 \mathrm{~K}$, which is supported by the expected saturation of $g(T)$ due to abovementioned QD size limit. An upper limit is obtained by extrapolating the square root fit to the $f_{\text {osc }}(T)$ dependence in Fig. 4 (black solid line) beyond $35 \mathrm{~K}$. In this way the increase of the OS with temperature is likely to be overestimated as the expected saturation is not accounted for because only experimental data points below saturation are extrapolated. The associated VRS calculated for a low temperature coupling constant equal to $56 \mu \mathrm{eV}$, a Q-factor of 13000 and QD sizes of 4.5, 6.0, and 7.5 nm is presented 


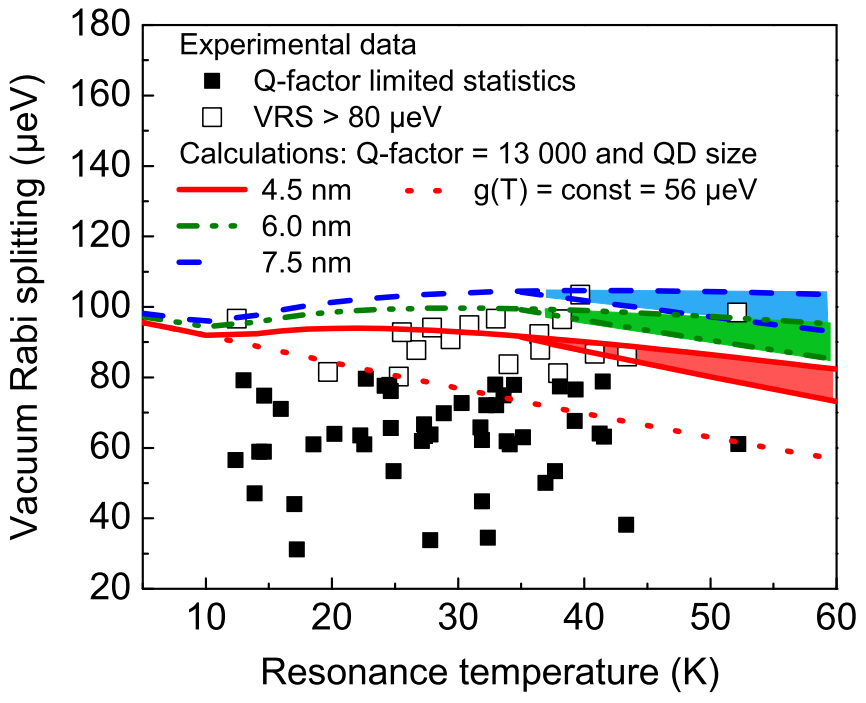

FIG. 7. (Color online) Comparison of the experimental dependence of the VRS on the resonance temperature (black squares, cases with VRS exceeding $80 \mu \mathrm{eV}$ marked with empty symbols) with theoretical calculations of acoustic phonon-induced changes of the VRS for a cavity with Q-factor equal to 13000 and QD size of $4.5 \mathrm{~nm}$ with (red solid line) and without (red dotted line) temperature dependence of the light-matter coupling constant included. For the case of a temperature-dependent coupling constant calculations for QD sizes of $6.0 \mathrm{~nm}$ (green dash-dotted line) and $7.0 \mathrm{~nm}$ (dashed blue line) are also presented. For each QD size a lower [assuming $g=g(T=35 \mathrm{~K})=$ const $]$ and upper bound $[g(T)$ obtained by extrapolating the square root fit to the experimental $f_{\text {osc }}(T)$ dependence in Fig. 4] of the temperature dependence of the VRS above $35 \mathrm{~K}$ is presented.

in Fig. 7. For comparison the red dotted line also shows the simulated temperature dependence of the VRS for a constant value of $g$ for the 4.5-nm-sized QD as it is expected for typical strongly confined QDs. Indeed, in agreement with our experimental observations, the increase of the OS strength overcompensates the temperature-induced decrease of the VRS due to the phonon coupling, yielding a roughly constant value of the VRS. Although for higher temperatures the experimental temperature-dependent light-matter coupling strength is not available in the present experiment (Fig. 4), it is clear that at some point $g$ will saturate and therefore for very high temperatures the VRS is expected to decrease due to the dominating phonon-induced dephasing. As shown in Fig. 7 the difference between the upper and lower bound of the VRS dependence on temperature for a given QD size is limited to $10 \%$ range only. When making a comparison between the calculations and the experimentally observed statistical ensemble data one has to keep in mind that the variations of the light-matter coupling strength that are due to the random positions of the QDs inside the micropillars are not included in the simulations and we therefore have to concentrate on the QDs that are positioned close to the center of the cavity where the coupling strength is maximal, as described previously. To this end we selected the strong coupling cases with a VRS exceeding $80 \mu \mathrm{eV}$, which is the minimal VRS that is estimated based on the mode volume and the OS when disregarding all loss channels and assuming perfect spectral and spatial matching of the QD to the cavity mode, and marked them with empty symbols in Fig. 7. For this subset of the experimental data the VRS is increasing with $55.5 \%$ probability. The reduction of the probability for an increasing trend is related to the strong scattering of the experimental data and the reduced number of data points which impairs the significance of the statistics. The envelope of the data points shows a behavior similar to the temperature dependence expected from the theory when the temperature dependence of the light-matter coupling constant is included in the calculations.

The underlying effect of the temperature-induced change of the state coupling most efficiently to the CM towards substates with larger wave function extension is an inherent property of large QDs and makes these systems very exceptional and attractive. Not only does it allow for the realization of the strong coupling regime on the single $\mathrm{X}$-single photon level, but also forces back phonon-induced decoherence by compensating their detrimental influence. This effect has been experimentally observed in a solid state system and can pave the way for applications of strongly coupled systems at elevated temperatures.

\section{SUMMARY}

We have studied, both theoretically and experimentally, the temperature dependence of the vacuum Rabi splitting. A statistical approach, enabled by the superb sample technology, has been utilized. It allowed for a temperature-dependent study on 89 strongly coupled QD-micropillars in a wide temperature range of (10-50) K. Our experimental results indicate an increase of the vacuum Rabi splitting with temperature. While for strongly confined QDs a decrease of the VRS with temperature due to the dephasing induced by acoustic phonons is expected, the large QDs studied here show a temperature-dependent light-matter coupling strength which seems to overcompensate the phonon effects in the intermediate temperature range. The strong influence of the wave function localization length in the growth direction, the light-matter coupling constant, and the cavity losses on the initial (low-temperature) value of the VRS as well as its renormalization have been addressed theoretically. The positive temperature coefficient of the VRS is an inherent and unique feature of large QDs, which has been proven to maintain the strong coupling in QD-micropillars up to temperatures as high as $50 \mathrm{~K}$. This shows that complex electronic structures, like the large QDs discussed here, combining two-level system behavior with additional properties, can be very advantageous for $\mathrm{CQED}$ and possible applications in quantum information technology.

\section{ACKNOWLEDGMENTS}

The authors acknowledge financial support from Deutsche Forschungsgemeinschaft (DFG) via projects AX17/7-1 and RE2974/5-1. A.M. appreciates support from the Polish Ministry of Science and Higher Education within the "Mobilność Plus" programme. We thank M. Emmerling and A. Wolf for expert sample preparation. 
[1] J. P. Reithmaier, G. Sęk, A. Löffler, C. Hofmann, S. Kuhn, S. Reitzenstein, L. V. Keldysh, V. D. Kulakovskii, T. L. Reinecke, and A. Forchel, Strong coupling in a single quantum dotsemiconductor microcavity system, Nature (London) 432, 197 (2004).

[2] T. Yoshie, A. Scherer, J. Hendrickson, G. Khitrova, H. M. Gibbs, G. Rupper, C. Ell, O. B. Shchekin, and D. G. Deppe, Vacuum Rabi splitting with a single quantum dot in a photonic crystal nanocavity, Nature (London) 432, 200 (2004).

[3] S. Haroche and J. Raimond, Exploring the Quantum (Oxford University Press, Oxford, 2006).

[4] J. I. Cirac, P. Zoller, H. J. Kimble, and H. Mabuchi, Quantum State Transfer and Entanglement Distribution among Distant Nodes in a Quantum Network, Phys. Rev. Lett. 78, 3221 (1997).

[5] A. Imamoglu, D. D. Awschalom, G. Burkard, D. P. DiVincenzo, D. Loss, M. Sherwin, and A. Small, Quantum Information Processing Using Quantum Dot Spins and Cavity QED, Phys. Rev. Lett. 83, 4204 (1999).

[6] M. A. Nielsen and I. L. Chuang, Quantum Computation and Quantum Information (Cambridge University Press, Cambridge, 2000).

[7] S. Reitzenstein, C. Hofmann, A. Gorbunov, M. Strauß, S. H. Kwon, C. Schneider, A. Löffler, S. Höfling, M. Kamp, and A. Forchel, AlAs/GaAs micropillar cavities with quality factors exceeding 150.000, Appl. Phys. Lett. 90, 251109 (2007).

[8] K. Aoki, D. Guimard, M. Nishioka, M. Nomura, S. Iwamoto, and Y. Arakawa, Coupling of quantum-dot light emission with a three-dimensional photonic-crystal nanocavity, Nat. Photon. 2, 688 (2008).

[9] I. Wilson-Rae and A. Imamoglu, Quantum dot cavity-QED in the presence of strong electron-phonon interactions, Phys. Rev. B 65, 235311 (2002).

[10] F. Milde, A. Knorr, and S. Hughes, Role of electron-phonon scattering on the vacuum Rabi splitting of a single-quantum dot and a photonic crystal nanocavity, Phys. Rev. B 78, 035330 (2008).

[11] G. Tarel and V. Savona, Emission spectrum of a quantum dot embedded in a nanocavity, Phys. Status Solidi C 6, 902 (2009).

[12] M.Glässl, L. Sörgel, A. Vagov, M. D. Croitoru, T. Kuhn, and V. M. Axt, Interaction of a quantum-dot cavity system with acoustic phonons: Stronger light-matter coupling can reduce the visibility of strong coupling effects, Phys. Rev. B 86, 035319 (2012).

[13] D. P. S. McCutcheon and A. Nazir, Model of the Optical Emission of a Driven Semiconductor Quantum Dot: PhononEnhanced Coherent Scattering and Off-Resonant Sideband Narrowing, Phys. Rev. Lett. 110, 217401 (2013).

[14] P. Kaer and J. Mørk, Decoherence in semiconductor cavity QED systems due to phonon couplings, Phys. Rev. B 90, 035312 (2014).

[15] C. Roy and S. Hughes, Phonon-Dressed Mollow Triplet in the Regime of Cavity Quantum Electrodynamics: ExcitationInduced Dephasing and Nonperturbative Cavity Feeding Effects, Phys. Rev. Lett. 106, 247403 (2011).

[16] Z. Harsij, M. Bagheri Harouni, R. Roknizadeh, and M. H. Naderi, Influence of electron-phonon interaction on the optical spectrum and quantum statistics in a quantum-dot-cavity system: Master-equation approach, Phys. Rev. A 86, 063803 (2012).
[17] Y. Ota, S. Iwamoto, N. Kumagai, and Y. Arakawa, Impact of electron-phonon interactions on quantum-dot cavity quantum electrodynamics, arXiv:0908.0788.

[18] K. Müller, K. A. Fischer, A. Rundquist, C. Dory, K. G. Lagoudakis, T. Sarmiento, Y. A. Kelaita, V. Borish, and J. Vučković, Ultrafast Polariton-Phonon Dynamics of Strongly Coupled Quantum Dot-Nanocavity Systems, Phys. Rev. X 5, 031006 (2015).

[19] Y.-J. Wei, Y. He, Y.-M. He, C.-Y. Lu, J.-W. Pan, C. Schneider, M. Kamp, S. Höfling, D. P.S. McCutcheon, and A. Nazir, Temperature-Dependent Mollow Triplet Spectra from a Single Quantum Dot: Rabi Frequency Renormalization and Sideband Linewidth Insensitivity, Phys. Rev. Lett. 113, 097401 (2014).

[20] A. Vagov, M. D. Croitoru, M. Gläß1, V. M. Axt, and T. Kuhn, Real-time path integrals for quantum dots: Quantum dissipative dynamics with superohmic environment coupling, Phys. Rev. B 83, 094303 (2011).

[21] A. Vagov, M. Gläß1, M. D. Croitoru, V. M. Axt, and T. Kuhn, Competition between pure dephasing and photon losses in the dynamics of a dot-cavity system, Phys. Rev. B 90, 075309 (2014).

[22] S. Reitzenstein, S. Münch, P. Franeck, A. Rahimi-Iman, A. Löffler, S. Höfling, L. Worschech, and A. Forchel, Control of the Strong Light-Matter Interaction between an Elongated $\mathrm{In}_{0.3} \mathrm{Ga}_{0.7}$ As Quantum Dot and a Micropillar Cavity Using External Magnetic Fields, Phys. Rev. Lett. 103, 127401 (2009).

[23] D. Press, S. Götzinger, S. Reitzenstein, C. Hofmann, A. Löffler, M. Kamp, A. Forchel, and Y. Yamamoto, Photon Antibunching from a Single Quantum-Dot-Microcavity System in the Strong Coupling Regime, Phys. Rev. Lett. 98, 117402 (2007).

[24] J. Kasprzak, S. Reitzenstein, E. A. Muljarov, C. Kistner, C. Schneider, M. Strauss, S. Höfling, A. Forchel, and W. Langbein, Up on the Jaynes-Cummings ladder of a quantumdot/microcavity system, Nat. Mater. 9, 304 (2010).

[25] F. Albert, K. Sivalertporn, J. Kasprzak, M. Strauß, C. Schneider, S. Höfling, M. Kamp, A. Forchel, S. Reitzenstein, E.A. Muljarov, and W. Langbein, Microcavity controlled coupling of excitonic qubits, Nat. Commun. 4, 1747 (2013).

[26] A. Löffler, J. P. Reithmaier, A. Forchel, A. Sauerwald, D. Peskes, T. Kümmell, and G. Bacher, Influence of the strain on the formation of GaInAs/GaAs quantum structures, J. Cryst. Growth 286, 6 (2006).

[27] M. Syperek, M. Baranowski, G. Sęk, J. Misiewicz, A. Löffler, S. Höfling, S. Reitzenstein, M. Kamp, and A. Forchel, Impact of wetting-layer density of states on the carrier relaxation process in low indium content self-assembled (In,Ga)As/GaAs quantum dots, Phys. Rev. B 87, 125305 (2013).

[28] L. C. Andreani, G. Panzarini, and J.-M. Gérard, Strong-coupling regime for quantum boxes in pillar microcavities: Theory, Phys. Rev. B 60, 13276 (1999).

[29] A. Musiał, P. Gold, J. Andrzejewski, A. Löffler, J. Misiewicz, S. Höfling, A. Forchel, M. Kamp, G. Sęk, and S. Reitzenstein, Toward weak confinement regime in epitaxial nanostructures: Interdependence of spatial character of quantum confinement and wave function extension in large and elongated quantum dots, Phys. Rev. B 90, 045430 (2014).

[30] B. Krummheuer, V. M. Axt, and T. Kuhn, Theory of pure dephasing and the resulting absorption line shape in semiconductor quantum dots, Phys. Rev. B 65, 195313 (2002). 
[31] A. Grodecka, L. Jacak, P. Machnikowski, and K. Roszak, Quantum Dots: Research Developments, edited by P. A. Ling (Nova Science, New York, 2005), p. 47.

[32] S. Reitzenstein, C. Böckler, A. Löffler, S. Höfling, L. Worschech, A. Forchel, P. Yao, and S. Hughes, Polarizationdependent strong coupling in elliptical high-Q micropillar cavities, Phys. Rev. B 82, 235313 (2010).

[33] S. Ohno, S. Adachi, R. Kaji, S. Muto and H. Sasakura, Optical anisotropy and photoluminescence polarization in single InAlAs quantum dots, Appl. Phys. Lett. 98, 161912 (2011).

[34] S. Reitzenstein and A. Forchel, Quantum dot micropillars, J. Phys. D: Appl. Phys. 43, 033001 (2010).

[35] M. Bayer and A. Forchel, Temperature dependence of the exciton homogeneous linewidth in $\mathrm{In}_{0.60} \mathrm{Ga}_{0.40} \mathrm{As} / \mathrm{GaAs}$ self-assembled quantum dots, Phys. Rev. B 65, 041308(R) (2002).

[36] L. Besombes, K. Kheng, L. Marsal, and H. Mariette, Acoustic phonon broadening mechanism in single quantum dot emission, Phys. Rev. B 63, 155307 (2001).

[37] P. Borri, W. Langbein, U. Woggon, V. Stavarache, D. Reuter, and A. D. Wieck, Exciton dephasing via phonon interactions in InAs quantum dots: Dependence on quantum confinement, Phys. Rev. B 71, 115328 (2005).

[38] E. Peter, P. Senellart, D. Martrou, A. Lemaitre, J. Hours, J. M. Gerard, and J. Bloch, Exciton-Photon Strong-Coupling Regime for a Single Quantum Dot Embedded in a Microcavity, Phys. Rev. Lett. 95, 067401 (2005).

[39] P. Borri, W. Langbein, S. Schneider, U. Woggon, R. L. Sellin, D. Ouyang, and D. Bimberg, Ultralong Dephasing Time in InGaAs Quantum Dots, Phys. Rev. Lett. 87, 157401 (2001).

[40] F. Rossi and T. Kuhn, Theory of ultrafast phenomena in photoexcited semiconductors, Rev. Mod. Phys. 74, 895 (2002).

[41] A. Musiał, P. Kaczmarkiewicz, G. Sęk, P. Podemski, P. Machnikowski, J. Misiewicz, S. Hein, S. Höfling, and A. Forchel, Carrier trapping and luminescence polarization in quantum dashes, Phys. Rev. B 85, 035314 (2012).

[42] A. Vagov, V. M. Axt, T. Kuhn, W. Langbein, P. Borri, and U. Woggon, Nonmonotonous temperature dependence of the initial decoherence in quantum dots, Phys. Rev. B 70, 201305(R) (2004).

[43] J.H. Quilter, A. J. Brash, F. Liu, M. Glässl, A. M. Barth, V.M. Axt, A.J. Ramsay, M.S. Skolnick, and A. M. Fox, Phonon-Assisted Population Inversion of a Single InGaAs/GaAs Quantum Dot by Pulsed Laser Excitation, Phys. Rev. Lett. 114, 137401 (2015).

[44] S. Bounouar, M. Müller, A. M. Barth, M. Glässl, V. M. Axt, and P. Michler, Phonon-assisted robust and deterministic twophoton biexciton preparation in a quantum dot, Phys. Rev. B 91, 161302(R) (2015). 